\title{
Osteoarthritis at Young Age, a Diagnostic Challenge: A Case of Stickler Syndrome
}

\author{
Isabelle de Wergifosse ${ }^{*}$ and Rene Westhovens
}

Division of Rheumatology, University Hospital, Leuven, Belgium

\begin{abstract}
A young woman presents with severe polyarticular osteoarthritis with relevant family history potentially suggesting a hereditary disease. Previously, the patient's mother had been diagnosed with rheumatoid arthritis and reported to have suffered from some locomotor problems. Careful clinical evaluation with an extensive personal and familial history pointed towards a diagnosis of Stickler syndrome, an autosomal dominant condition with progressive arthro-ophthalmopathy, (early osteoarthritis and myopia). Following this timely diagnosis, genetic counselling was offered.
\end{abstract}

Keywords: Myopia, osteoarthritis, scoliosis, Stickler Syndrome.

\section{INTRODUCTION}

Complete and timely medical diagnoses rely on the identification of symptom patterns that are characteristic for certain patient demographics. Therefore, it is plausible that a disease presenting at an unusual age might be overlooked as part of the differential diagnosis. Furthermore, a disease commonly associated with the elderly that presents at a younger age might indicate a hereditary disease; in which a diagnosis is a priority for genetic counselling. Here, we report a patient presenting with early onset polyarticular osteoarthritis, with a potential hereditary cause. A full clinical evaluation and an extensive personal and familial history taking resulted in the correct diagnosis. The patient gave oral informed consent for this report.

\section{CASE REPORT}

A 26-year old female patient presents with arthralgia and intermittent swelling of the PIP joints, ankles and knees of several years. Symptoms worsen on joint loading and when participating in sports, there was no morning stiffness or pain at night. A brief medical history shows that she underwent a fusion procedure for scoliosis and a Ravitch procedure for pectus carinatum during childhood. She suffered a hip fracture aged 18 and was previously diagnosed with myopia. Clinical examination revealed a woman with short stature $(1.60 \mathrm{~m})$ with exceptionally long and thin fingers, a status post scoliosis fusion and chest surgery, and mild hydrops with flexion deficit in the right knee. Applying patellar pressure was painful for both knees. Radiology revealed significant degenerative changes of the knees, ankles and some PIP joints (Figs. 1, 2).

*Address correspondence to this author at the Division of Rheumatology, University Hospital, Leuven, Belgium; Tel: 016-342541; Fax: 016-342543;

E-mail: isabelle.dewergifosse@uzleuven.be, isabelleke424@msn.com

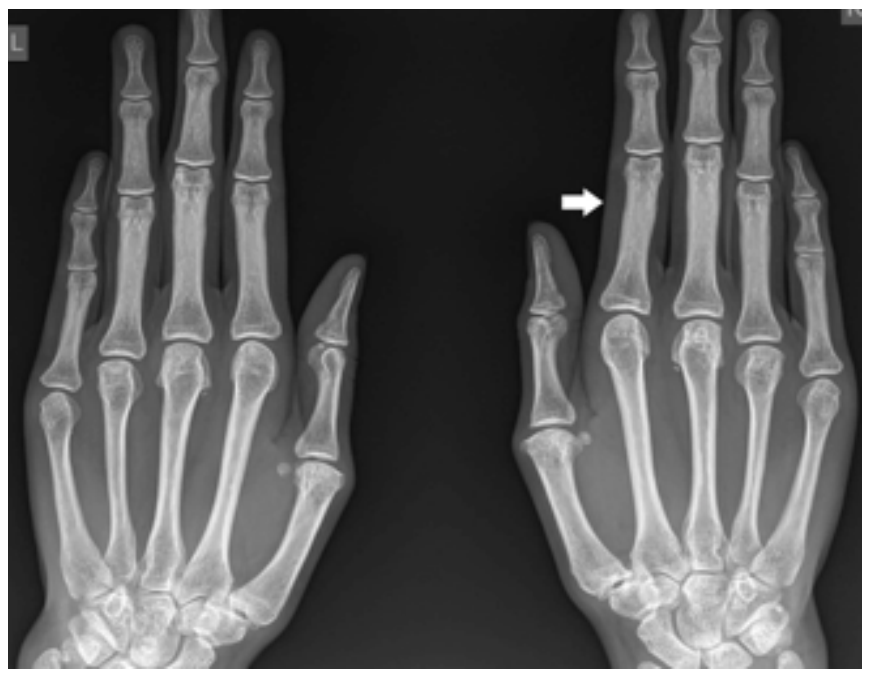

Fig. (1). X-rays showing the patient's unusually slender hands and long phalanges (arrow), a feature that can be seen in patients with Stickler syndrome.

The patient was accompanied to the first outpatient visit by her mother, who had previously undergone a bilateral hip replacement in her thirties. The patient's mother was also treated for presumed rheumatoid factor negative rheumatoid arthritis (RA). The patient's grandfather also suffered from "early arthrosis". A new radiological evaluation was requested from the rheumatologist overseeing the mother's follow up care. Results from this clearly showed polyarticular osteoarthritis with no typical RA erosions (Figs. 3, 4).

The main reason for this young female to attend a consultation was her concern about an inheritable disease. The combination of familial osteoarthritis at a young age, early scoliosis and the myopia suggests a diagnosis of autosomal dominant Stickler syndrome; in depth analysis revealed a c. $2155 \mathrm{C}>\mathrm{T}$ mutation in the COL2A1 gene corresponding with Stickler syndrome. 


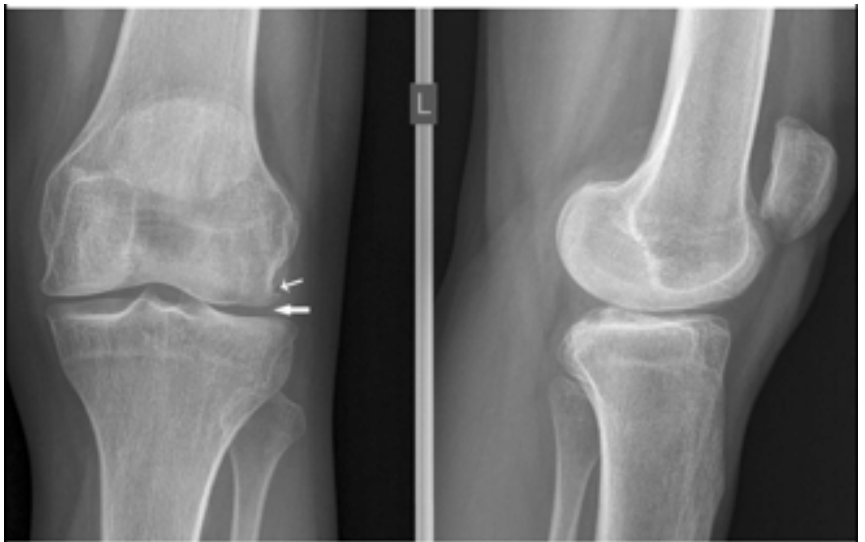

Fig. (2). X-rays of the patient's knees showing signs of osteoarthritis, the broad arrow indicating marked joint space narrowing. The small arrow indicates an osteophyte.

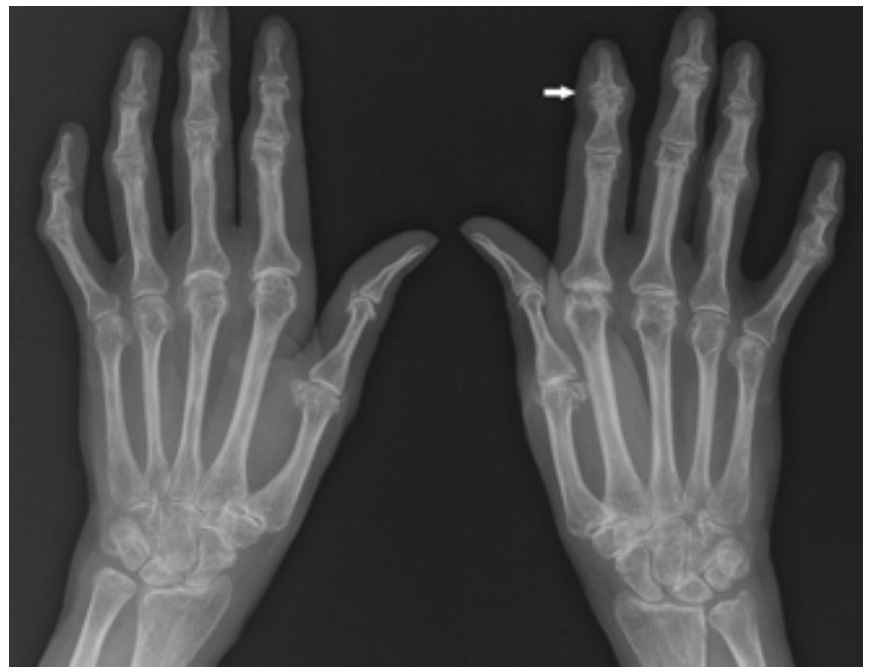

Fig. (3). X-rays of the mothers' hands showing signs of osteoarthritis. We see joint space narrowing in most of the MCP, PIP and DIP joints, accompanied by osteophyte formation.. These features, with the absence of erosive disease and involvement of the DIP joints, are not consistent with a diagnosis of rheumatoid arthritis.

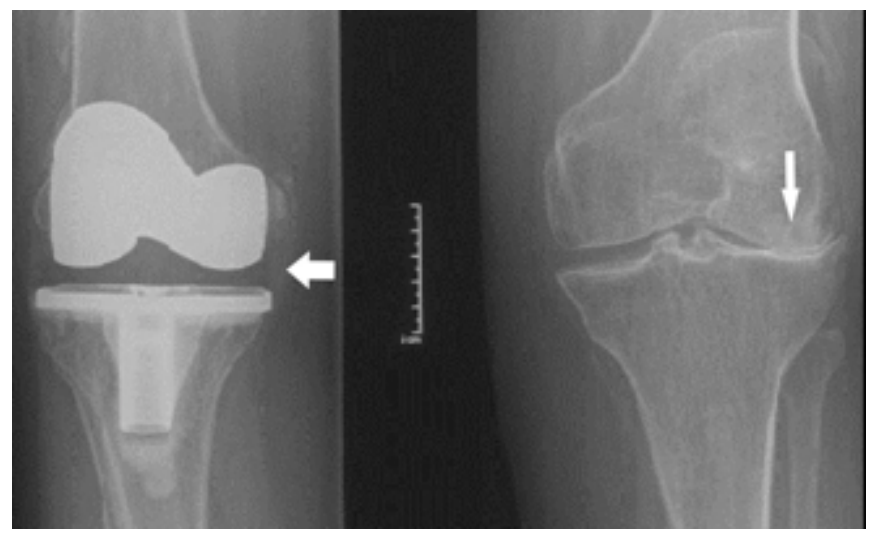

Fig. (4). X-rays of the mothers' knees showing right knee replacement (broad arrow) and left-sided severe osteoarthritis marked by extreme lateral joint space narrowing with valgus (small arrow).

\section{DISCUSSION}

Gunnar B. Stickler first described Stickler syndrome in 1965 as an autosomal dominant progressive arthroophthalmopathy [1]. Disease manifestations are variable with a late or misdiagnosis being common. Clinical features consist of orofacial and ophthalmic features, deafness and osteoarthritis. Typical orofacial features include a flat midface with a depressed nasal bridge, anteverted nares and micrognathia [1-4]. The majority of families with Stickler syndrome have mutations in the COL2A1 gene: Type 1 or membranous-vitreous type. Others may have mutations in the COL11A1 gene, known as Type 2. Mutations in COL11A2 have also been described, displaying a more systemic syndrome but no ophthalmological features, known as Type 3. Other loci are yet to be identified. Osteoarticular manifestations are common and include early-onset osteoarthritis, spinal abnormalities and joint hypermobility, with up to $50 \%$ of patients developing osteoarthritis before the age of 30 [5]. The typical orofacial features were not present in this patient, although these can become less expressive with increasing age, and the patient suffered no hearing loss. The slender fingers seen here are infrequently reported in literature. In summary, our patient was suffering from early osteoarthritis in the knee joints, like her mother who had previously underwent replacement knee surgery. There was no valgus component which is often reported. As osteoarthritis in Stickler syndrome is more frequently seen in weight-bearing joints like the hips or knee, this, coupled to the other skeletal features of a pectus carinatum and scoliosis and the myopia were compatible with a diagnosis of Stickler syndrome [6].

This case report highlights that detailed personal and familial history and full clinical evaluation are crucial to making diagnosis in patients with unusual symptom presentation. The importance of timely diagnoses for hereditary disorders, cannot be emphasised enough to ensure adequate genetic counselling.

Furthermore, when reviewing the patient's file from the orthopaedic department at the time of scoliosis surgery, it was discovered that a diagnosis of Stickler syndrome had been suggested, although no diagnostic confirmation was carried out. Despite this, the patient, her mother nor the patient's GP were aware of this syndrome. This highlights the importance of proper transition of youngsters with serious diseases from paediatric into adult care.

\section{ABBREVIATIONS}

$\begin{array}{lll}\text { DIP } & = & \text { Distal Interphalangeal } \\ \text { GP } & = & \text { General Practitioner } \\ \text { MCP } & = & \text { Metacarpophalangeal } \\ \text { PIP } & = & \text { Proximal Interphalangeal } \\ \text { RA } & = & \text { Rheumatoid Arthritis }\end{array}$

\section{CONFLICT OF INTEREST}

The authors confirm that this article content has no conflict of interest. 


\section{ACKNOWLEDGEMENTS}

We would like to express our gratitude to the patient for letting us use this medical information.

\section{REFERENCES}

[1] Stickler GB, Belau PG, Farrell FJ, et al. Hereditary Progressive Arthro-Ophthalmopathy. Mayo Clin Proc 196; 40(6): 433-55.

[2] Snead MP, Yates JR. Clinical and Molecular genetics of Stickler syndrome. J Med Genet 1999; 36(5): 353-9.
[3] Rai A, Wordsworth P, Coppock JS, Zaphiropoulos GC, Struthers GR. Hereditary arthro-ophthalmopathy (Stickler syndrome): a diagnosis to consider in familial premature osteoarthritis. Br $\mathrm{J}$ Rheumatol 1994; 33(12): 1175-80.

[4] Temple IK. Stickler's syndrome. J Med Genet 1989; 26(2): 119-26.

[5] Couchouron T, Masson C. Early-onset progressive osteoarthritis with hereditary progressive ophtalmopathy or Stickler syndrome. Joint Bone Spine 2011; 78(1): 45-9. doi: 10.1016/j.jbspin.2010.03.012

[6] Rose PS, Ahn NU, Levy HP, et al. Thoracolumbar spinal abnormalities in Stickler syndrome. Spine (Phila Pa 1976). 2001; 26(4): 403-9.

(C) de Wergifosse and Westhovens; Licensee Bentham Open

This is an open access article licensed under the terms of the Creative Commons Attribution Non-Commercial License (http://creativecommons.org/licenses/by-nc/

3.0/) which permits unrestricted, non-commercial use, distribution and reproduction in any medium, provided the work is properly cited. 